\title{
SEARCH FOR DOUBLE DEGENERATE PROGENITORS OF SUPERNOVAE TYPE IA WITH SPY
}

\author{
R. NAPIWOTZKI, H. DRECHSEL, U. HEBER, C. KARL AND \\ E.-M. PAULI \\ Dr. Remeis-Sternwarte, Astronom. Institut, Universität \\ Erlangen-Nürnberg, Sternwartstr. 7, 96049 Bamberg, Germany \\ N. CHRISTLIEB, H.-J. HAGEN AND D. REIMERS \\ Hamburger Sternwarte, Universität Hamburg, Gojenbergsweg \\ 112, 21029 Hamburg, Germany \\ D. KOESTER AND S. MOEHLER \\ Institut für Theoretische Physik und Astrophysik, Universität \\ Kiel, 24098 Kiel, Germany \\ D. HOMEIER \\ Department of Physics \&3 Astronomy, University of Georgia, \\ Athens, GA 30602-2451, USA \\ B. LEIBUNDGUT AND A. RENZINI \\ European Southern Observatory, Karl-Schwarzschild-Str. 2, \\ 85748 Garching, Germany \\ T.R. MARSH \\ University of Southampton, Department of Physics \& \\ Astronomy, Highfield, Southampton S017 1BJ, UK \\ G. NELEMANS \\ Institute of Astronomy, Madingley Road, Cambridge \\ CB3 OHA, UK \\ AND \\ L. YUNGELSON \\ Institute of Astronomy of the Russian Academy of Sciences, \\ 48 Pyatnitskaya Str., 109017 Moscow, Russia
}

\begin{abstract}
We report on a large survey for double degenerate (DD) binaries as potential progenitors of type Ia supernovae with the UVES spectrograph at the ESO VLT (ESO SN Ia Progenitor surveY - SPY).
\end{abstract}


Supernovae of type Ia (SN Ia) play an outstanding role for our understanding of galactic evolution and the determination of the extragalactic distance scale. However, the nature of their progenitors is still unknown (e.g. Livio 2000). In the so-called double degenerate (DD) scenario (Iben \& Tutukov 1984) two white dwarfs with a mass exceeding the Chandrasekhar limit merge. Several systematic radial velocity $(\mathbf{R V})$ searches for DDs have been undertaken starting in the mid 1980's checking a total of $\approx 200$ white dwarfs RV for variations (cf. Marsh 2000 and references therein), but have failed to reveal any massive, short-period DD progenitor of SN Ia. However, this is not unexpected, as theoretical simulations suggest that only a few percent of all DDs are potential SN Ia progenitors (Iben, Tutukov \& Yungelson 1997; Nelemans et al. 2001).

In order to perform a definitive test of the DD scenario we have embarked on a large spectroscopic survey of 1500 white dwarfs (ESO SN Ia Progenitor surveY - SPY). SPY will overcome the main limitation of all efforts so far to detect DDs that are plausible SN Ia precursors: the samples of surveyed objects were too small. Spectra were taken with the highresolution UV-Visual Echelle Spectrograph (UVES) of the UT2 telescope (Kueyen) of the ESO VLT in service mode. Our instrument setup provides nearly complete spectral coverage from $3200 \AA$ to $6650 \AA$ with a resolution $R=18500(0.36 \AA$ at $\mathrm{H} \alpha)$. Due to the nature of the project, two spectra at different, "random" epochs separated by at least one day are observed.

ESO provides a data reduction pipeline for UVES, which formed the basis for our first selection of DD candidates. A careful re-reduction of the spectra is in progress. Differing from previous surveys we use a correlation procedure to determine RV shifts of the observed spectra (cf. Napiwotzki et al. 2001a). We routinely measure RVs with an accuracy of $\approx 2 \mathrm{~km} \mathrm{~s}^{-1}$ or better, therefore running only a very small risk of missing a merger precursor, which have orbital velocities of $150 \mathrm{~km} \mathrm{~s}^{-1}$ or higher.

Results. We have analyzed spectra of 558 white dwarfs and pre-white dwarfs taken during the first two years of the SPY project and detected 90 new DDs, 13 are double-lined systems (only 6 were known before). SPY is the first RV survey which performs a systematic investigation of both classes of white dwarfs: DAs and non-DAs. Our observations have already increased the DD sample by a factor of five. After completion, a final sample of $\approx 200$ DDs is expected.

Follow-up observations of this sample are mandatory to determine periods and white dwarf parameters and find potential SN Ia progenitors among the candidates. Good statistics of a large DD sample will also set stringent constraints on the evolution of close binaries, which will dramatically improve our understanding of this phase of stellar evolution. Starting in 2001 
TABLE 1. Fraction of RV variable stars in the current SPY sample for different spectral classes. WD+dM denotes systems for which a previously unknown cool companion is evident from the red spectra (not included in the DA/non-DA entries).

\begin{tabular}{l|r|r|r} 
Spectral type & total & RV variable & detection rate \\
\hline All DDs & 558 & 90 & $16 \%$ \\
non-DA (DB,DO,DZ) & 72 & 10 & $14 \%$ \\
WD+dM & 30 & 14 & $47 \%$
\end{tabular}

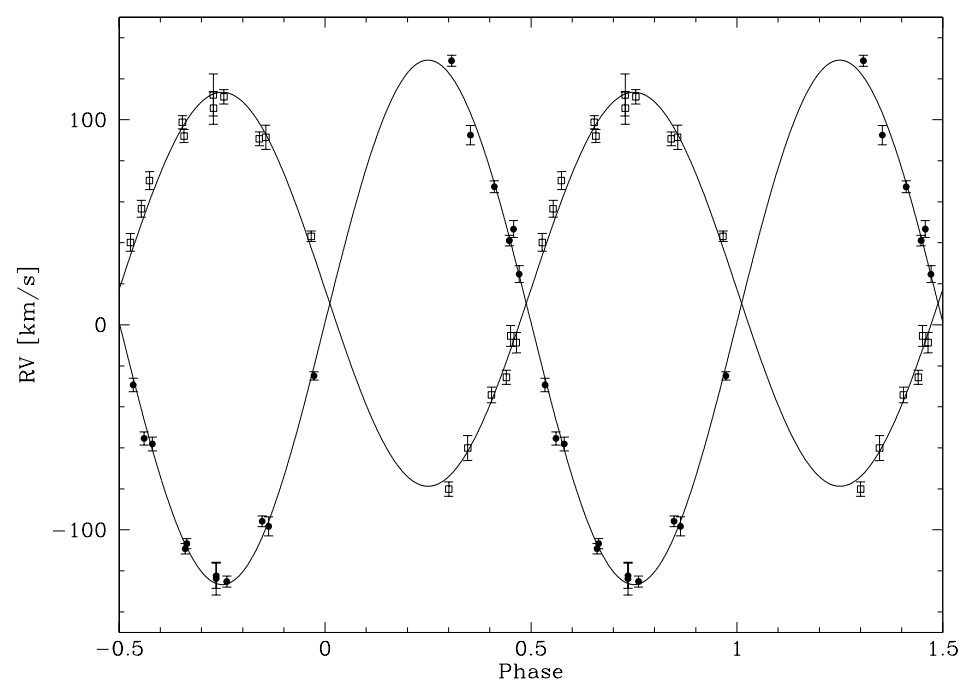

Figure 1. Measured RVs as a function of orbital phase and fitted sine curves for HE 1414-0848. Filled circles/open rectangles indicate the less/more massive components A and B. Note the difference of the "systemic velocities" $\gamma_{0}$ between both components caused by gravitational redshift.

follow-up observations have been carried out with VLT and NTT of ESO as well as with the $3.5 \mathrm{~m}$ telescope of the Calar Alto observatory/Spain and the INT (Napiwotzki et al. 2001a, 2001b, 2002; Karl et al., these proceedings). Our sample includes many short period binaries, several with masses closer to the Chandrasekhar limit than any system known before. During our follow-up observations we have detected a very promising potential SN Ia precursor candidate. However, some additional spectra are necessary to verify our RV curve solution. Results will be reported elsewhere.

Exemplary for other double-lined systems we discuss here the DA+DA system HE 1414-0848. The orbital period of $P=12^{\mathrm{h}} 25^{\mathrm{m}} 44^{\mathrm{s}}$ and semiamplitudes of $127 \mathrm{~km} \mathrm{~s}^{-1}$ and $96 \mathrm{~km} \mathrm{~s}^{-1}$ are derived for the individual components (Napiwotzki et al. 2002). RV curves for both components are displayed in Fig. 1. The ratio of velocity amplitudes is directly related to 
the mass ratio of both components. Additional information comes from the mass dependent gravitational redshift. The difference in gravitational redshift corresponds to the apparent difference of "systemic velocities" of both components, as derived from the RV curves (Fig. 1). Only one set of individual white dwarf masses fulfills the constraints given by both the amplitude ratio and redshift difference (for a given mass-radius relation). We estimate the masses of the individual components with this method to be $0.55 M_{\odot}$ and $0.71 M_{\odot}$ for $\mathrm{A}$ and $\mathrm{B}$, respectively. This translates into $\log g=7.92$ and 8.16, respectively.

Another estimate of the white dwarf parameters is available from a model atmosphere analysis of the combined spectrum. We have developed a new tool (FITSB2), which performs a spectral analysis of both components of a double-lined system. The fit is performed on all available spectra, covering different orbital phases simultaneously. We fitted temperatures and gravities of both components of HE 1414-0848 (the mass ratio fixed at the accurate value derived from the RV curve). The results are $T_{\text {eff }} / \log g=$ $8380 \mathrm{~K} / 7.83$ and $10900 \mathrm{~K} / 8.14$ for $\mathrm{A}$ and $\mathrm{B}$, which are in good agreement with the $\log g$ values predicted from the analysis of the RV curve. The total mass of the HE $1414-0848$ system is $1.26 M_{\odot}$, only $10 \%$ below the Chandrasekhar limit. The system will merge due to loss of angular momentum via gravitational wave radiation after two Hubble times.

Spin-off results. SPY produces an immense, unique sample of very high resolution white dwarf spectra. It will allow us for the first time to tackle many longstanding questions on a firm statistical basis. Among those are the mass distribution of white dwarfs (Koester et al. 2001), kinematical properties of the white dwarf population (Pauli, these proceedings), surface compositions, luminosity function, rotational velocities, and detection of weak magnetic fields. A more detailed description of ongoing spin-off activity is given in Napiwotzki et al. (2001a). Members of the community interested in spin-off opportunities are invited to participate in the exploitation of the SPY sample.

\section{References}

Iben, I.Jr., Tutukov, A.V.: 1984, ApJS 54, 335

Iben, I.Jr., Tutukov, A.V., Yungelson, L.R.: 1997, ApJ 475, 291

Koester, D., Napiwotzki, R., Christlieb, N., et al.: 2001, A\&A 378, 556

Livio, M. 2000: in "Type Ia Supernovae: Theory and Cosmology", Cambridge Univ. Press, p. 33

Marsh, T.R.: 2000, NewAR 44, 119

Napiwotzki, R., Christlieb, N., Drechsel, H., et al.: 2001a, AN 322, 401

Napiwotzki, R., Edelmann, H., Heber, U., et al.: 2001b, A\&A 378, L17

Napiwotzki, R., Koester, K., Nelemans, G., et al.: 2002, A\&A 386, 957

Nelemans, G., Yungelson, L.R., Portegies Zwart, S.F., Verbunt, F.: 2001, A\&A 365, 491 